\title{
RON ADELSMAN
}

ANDREW WHINSTON

\section{The equivalence of three social decision functions}

Revue française d'automatique, d'informatique et de recherche opérationnelle. Recherche opérationnelle, tome 11, no 3 (1977), p. 257-265.

$<$ http://www.numdam.org/item?id=RO_1977_11_3_257_0>

(C) AFCET, 1977, tous droits réservés.

L'accès aux archives de la revue « Revue française d'automatique, d'informatique et de recherche opérationnelle. Recherche opérationnelle » implique l'accord avec les conditions générales d'utilisation (http://www.numdam.org/ legal.php). Toute utilisation commerciale ou impression systématique est constitutive d'une infraction pénale. Toute copie ou impression de ce fichier doit contenir la présente mention de copyright.

\section{Numdam}

Article numérisé dans le cadre du programme

Numérisation de documents anciens mathématiques

http://www.numdam.org/ 


\title{
THE EQUIVALENCE OF THREE SOCIAL DECISION FUNCTIONS
}

\author{
by Ron Adelsman and Andrew Whinston $\left({ }^{2}\right)$
}

\begin{abstract}
This paper demonstrates that three of the basic approaches to the solution of the social choice problem are in fact equivalent to one another All will yield the same social decision functions, a winning set of permutations of the actions The Combinatorial Optimization criterion of Blin and Whinston is shown to be monotonically related to the Kemeny function criterion proposed by Levenghick The set covering formulation for the $l_{1}$ norm case devised by Merchant and Rao is also shown to be equivalent to the other two The geometrical aspect of the problem is also discussed and an example is provided
\end{abstract}

\section{INTRODUCTION}

Recently several authors have proposed methods for determining a social ordering of a set of alternatives based on individual pairwise ordering of the set In each case the author had a different motivation for developing the particular function, but in all cases it was shown that a relationship existed between majority voting and the resulting social order. In this paper we show that all these formulations of the problem lead to exactly the same social ordering of alternatives when the data on pairwise preference are identical.

\section{CONBINATORIAL OPTIMIZATION CRITERION}

The Combinatorial Optımization criterion function of Blın and Whinston [1] seeks to determine a best ranking of actions such that the sum of vote proportions of each action over those lower rankıng actions is maximized.

(1) This research was supported in part by National Science Foundation, grant number EN675-07845

Reçu juıllet 1976, revisé déc 1976

$\left({ }^{2}\right)$ Purdue University, Lafayette (Indiana) U S A

R A I R O Recherche Operationnelle/Operations Research, vol 11, n 3, août 1977 
This optimal assignment of actions to ranks is determined by a permutation on the original order of the actions $\left[a_{1}, a_{2}, \ldots, a_{m}\right]$ to get $\left[a_{p(1)}, a_{p(2)}, \ldots, a_{p(m)}\right]$, such that $p(i)=k$ implies that $a_{k}$ has $i-1$ actions considered superior to it.

Let $A=\left[a_{i j}\right]$ be the matrix of vote proportions such that :

$a_{i j} \equiv$ proportion of individuals who prefer $a_{i}$ to $a_{j}$

$a_{j i} \equiv$ proportion of individuals who prefer $a_{j}$ to $a_{i}$

$a_{i i} \equiv 0$ (rather than the alternative value $1 / 2$ )

The case of individuals who have $a_{i} \sim a_{j}$ (indifference) can be resolved by splitting the votes equally between the strict orderings.

Define $Q=\left[q_{i j}\right]$ as the appropriate summation matrix with :

$$
q_{i j}=\left\{\begin{array}{lll}
0 & \text { if } & 1 \geq J \\
1 & \text { if } & i<j
\end{array}\right.
$$

Then,

$$
\phi(\bar{p})=\operatorname{M}_{p} \underset{x}{ } A_{p} \cdot Q
$$

is the desired criterion function. where

$$
A_{p}=\left[a_{p(t) p(J)}\right] .
$$

Here $a_{p(i) p(j)} q_{i j}>0$ implies that the permutation $p$ has ranked action $i$ over action $l$, and the dot product of two matrices of the same dimension is defined as

$$
A_{p} \cdot Q=\sum_{\forall(i, j)} a_{p(i) p(j)} q_{i j}
$$

Since $p$ is a permutation we can define the corresponding permutation matrix $P_{p}=\left[p_{k j}\right]$ as

$$
p_{k j}= \begin{cases}1 & \text { if } a_{j} \text { has rank k } \\ 0 & \text { otherwise }\end{cases}
$$

Noll $1_{p}=P_{p} A P_{p^{\prime}}^{\prime}$ and $(1$.$) cull he cw1!len 10$ emphasize its Quadratic Assignment nature

$$
\phi(\bar{p})=\operatorname{Max}_{p} P_{p} A P_{p}^{\prime} \cdot Q .
$$

This criterion function seeks to determine the optimal assignment of actions to ranks, $\bar{p}$, over all feasible $m$ ! permutations of the $a_{i}$. 


\section{KEMENY FUNCTION}

The Kemeny function seeks to find those permutation points, $P^{r}$. that maximize a dot product with the translated election matrix $E_{d}$. Here a permutation point is defined as $P^{p}=P_{p}^{\prime} X P_{p}$ where $P_{p}$ is the same as before and

$$
X=\left[x_{i j}\right]=\left\{\begin{aligned}
+1 & \text { if } i<j \\
0 & \text { if } i=j \\
-1 & \text { if } i>j
\end{aligned}\right.
$$

The translated election matrix is defined to be in skew-symmetric form and is related to the previous election matrix $A$ by:

$$
E_{d}=A-A^{\prime} \text {. }
$$

As Levenglick has demonstrated $\left({ }^{1}\right)$, the Kemeny function equivalently seeks to determine the permutation point of minimum Euclidian distance from $E_{d}$, thus maximizing the total amount of agreement between $P^{p}$ and $E_{d}$. Equivalently, one can view the problem from the position of choosing the optimal permutation matrix in the following criterion function

$$
H\left(p^{*}\right)=\operatorname{Max}_{p} E_{d} \cdot P_{p}^{\prime} X P_{p} .
$$

As Levenglick has shown $\left({ }^{2}\right),(4)$ is extremely attractive in that it "is symmetric, faithful, equitable. Condorçet, consistent and continuous for all $m \geqslant 2$ ", and its consistent extension to the set of rationals on $\left(\begin{array}{c}m \\ 2\end{array}\right)$ space is the only function that satisfies all the above properties.

THEOREM 1. The criterion function (4) is an equivalent representation of (2), and hence the Combinatorial Optimization criterion also has the above fairness properties.

Proof. By equivalence is meant that if $\phi\left(p_{1}\right) \geqslant \phi\left(p_{2}\right)$ then $H\left(p_{1}\right) \geqslant H\left(p_{2}\right)$ and vice versa. Thus equivalence implies $\{\bar{p}\}=\left\{p^{*}\right\}$; the same ranking of actions optimizes both criteria. The set notation is used since it is possible to have multiple social optima, which results when more than one permutation satisfies (2) and (4). To establish the equivalence we shall introduce the column vector of $m$ ones labelled $e$. Furthermore, define

$$
Z=\left[z_{i j}\right]=e e^{\prime}-I= \begin{cases}0 & \text { if } i=j \\ 1 & \text { otherwise }\end{cases}
$$

Now, $A$ was defined so that

$$
A+A^{\prime}=Z
$$

(1) [5], Theorem 4, p. 41.

(') [5], Theorems 5 and 7, pp. 41-44.

vol. 11, n 3, août 1977 
Conditions (3) and (5) together imply

$$
E_{d}=2 A-Z \text {. }
$$

Similarly, $X=Q-Q^{\prime}$ and $Z=Q+Q^{\prime}$ imply

$$
X=2 Q-Z \text {. }
$$

It is easily seen that

$$
P_{p}^{\prime} Z P_{p}=P_{p} Z P_{p}^{\prime}=Z
$$

The dot product operation in (4) is invariant to any reordering $T$ ( ) of $E_{d}$ and $P_{p}^{\prime} X . P_{p}$ that preserves the matchup of their respective matrix entries. That is

$$
E_{d} \cdot P_{p}^{\prime} X P_{p}=T\left(E_{d}\right) \cdot T\left(P_{p}^{\prime} X P_{p}\right)
$$

for all such valid $T$.

$T$ ( ) can be considered to be the set of all possible permutations of the elements of matrix ( ).

$$
T()=P_{p}() P_{p}^{\prime}
$$

is a valid form-preserving manipulation.

Now, (4), (6), (7), (8) and (9) combine to yield:

$$
\begin{aligned}
E_{d} . P_{p}^{\prime} X P_{p} & =P_{p} E_{d} P_{p}^{\prime} \cdot P_{p} P_{p}^{\prime} X P_{p} P_{p}^{\prime} \\
& =\left(2 P_{p} A P_{p}^{\prime}-Z\right) \cdot(2 Q-Z) \\
& =4 P_{p} A P_{p}^{\prime} \cdot Q-2 P_{p} A P_{p}^{\prime} \cdot Z-2 Z \cdot Q+Z \cdot Z
\end{aligned}
$$

Since

then

$$
2 P_{p} A P_{p}^{\prime} \cdot Z=2 Z \cdot Q=Z \cdot Z=m(m-1),
$$

$$
E_{d} \cdot P_{p}^{\prime} X P_{p}=4 P_{p} A P_{p}^{\prime} \cdot Q-m(m-1) ;
$$

hence (4) and (2) are equivalent.

\section{GEOMETRICAL INTERPRETATION}

As Blin and Whinston showed $\left({ }^{3}\right)$, whenever majority voting yields a transitive social ordering of the $a_{i}$, the associated permutation matrix will be optimal for (2). Correspondingly, the permutation point $P^{p *}$ would be a matrix of +1 's, -1 's, and 0 's with:

$$
P_{i j}^{p *}=\left\{\begin{array}{l}
0 \text { if } i=j \\
+1 \text { if } a_{i} \text { is preferred to } a_{j} \text { in the optimal social order } \\
-1 \text { if } a_{j} \text { is preferred to } a_{i} \text { in the optimal social order }
\end{array}\right.
$$

$\left({ }^{3}\right)[1]$ 
$p^{*}$ would be optimal for

$$
H\left(p^{*}\right)=\operatorname{Max}_{p} E_{d} \quad P^{p}
$$

Let us define $F$ as the set of all permutation points and $\operatorname{sgn}\left(E_{d}\right)$ as a matrix of +1 's, -1 's, and 0 's, whose entries correspond to the sign of the entries of $E_{d}$ Then it is clear that if $\operatorname{sgn}\left(E_{d}\right) \in F$, then $\operatorname{sgn}\left(E_{d}\right)=P^{p}$ and majority voting has yielded a transitive social ordering

Thus, we can rewrite $(4)^{\prime}$ as the following equivalent problem

$$
H\left(p^{*}\right)=-\operatorname{Min} E_{d}\left[\operatorname{sgn}\left(E_{d}\right)-P^{p}\right]+E_{d} \operatorname{sgn}\left(E_{d}\right)
$$

Since $Z \operatorname{sgn}\left(E_{d}\right)=Z \quad P^{p}=0$, (13) is equivalent to (14), using (6)

$$
H\left(p^{*}\right)=-2 \operatorname{Min} A \quad\left[\operatorname{sgn}\left(E_{d}\right)-P^{p}\right]+E_{d} \operatorname{sgn}\left(E_{d}\right)
$$

Thus, if $\operatorname{sgn}\left(E_{d}\right) \notin F$, we seek a permutation point that will entail a least cost for moving from an intransitive majority solution $\operatorname{sgn}\left(E_{d}\right)$ to a transitive social ordering given by $P^{p}$ In a geometric sense, $\operatorname{sgn}\left(E_{d}\right)$ and $P^{p}$ are vertices of a hypercube in $\left(\begin{array}{c}m \\ 2\end{array}\right)$ space, centered about the origin, with edge length of two The problem is to choose the closest vertex to sgn $\left(E_{d}\right)$ that belongs to $F$, where the measurement of distance is conditional upon $A$

The following lemma gives necessary and sufficient conditions for determining whether or not $\operatorname{sgn}\left(E_{d}\right) \in F$

Lemma Let $\operatorname{sgn}\left(E_{d}\right)=\left[\sigma_{\imath \jmath}\right]$ If there exist distinct $l, 1, k$ such that $\operatorname{sgn}\left(\sigma_{\imath \jmath}\right)=\operatorname{sgn}\left(\sigma_{\jmath^{k}}\right) \neq \operatorname{sgn}\left(\sigma_{\imath k}\right)$ then $\operatorname{sgn}\left(E_{d}\right) \notin F$ If no such $l, 1, k$ exist then $\operatorname{sgn}\left(E_{d}\right) \in F$

Proof If $\operatorname{sgn}\left(\sigma_{\imath \jmath}\right)=\operatorname{sgn}\left(\sigma_{\jmath k}\right) \neq \operatorname{sgn}\left(\sigma_{\imath k}\right)$, then either $\sigma_{\imath \jmath}=\sigma_{\jmath k}=+1$ or -1 In the former case, $\sigma_{\imath \jmath}=+1$ implies $a_{\imath \jmath}-a_{j}>0$, which in turn means that $a_{\imath}$ is preferred to $a_{\jmath}$ by majority voting Thus, $\sigma_{\imath \jmath}=\sigma_{\jmath k}=+1$ implies

$a_{\imath}$ is preferred to $a_{\jmath}$ is preferred to $a_{k}$, but $a_{k}$ is preferred to $a_{\imath}$

by majority voting, which is an intransitive ordering Thus, sgn $\left(E_{d}\right) \notin F$ In the latter case, the proof follows analogously

The above establishes sufficiency, necessity is now shown Assume that no such $l, 1, k$ exist that provide intransitivity Since all higher order intransitivities $\left({ }^{4}\right)$ require an intransitivity of triplets [2], there must exist a transitive ordering of the $a_{\imath}$ provided by sgn $\left(E_{d}\right)$, hence majority voting is transitive and optimal, and $\operatorname{sgn}\left(E_{d}\right) \in F$

(4) A $t^{\text {th }}$ order intransitivity is characterized as $a_{t_{1}}>a_{t_{2}} a_{t_{2}}>a_{t_{3}} \quad a_{t_{1}}>a$ but $a_{i t}>a_{i 1}$ 
In graph-theoretic terms, if one places a directed arc from node $i$ to $/$ to indicate $a_{\imath}$ ranked over $a_{j}$, then $\operatorname{sgn}\left(E_{d}\right) \in F$ if and only if there are no directed cycles within the graph. Furthermore, if there are directed cycles, an attempt to eliminate them by determing whether vertices of the hypercube adjacent to $\operatorname{sgn}\left(E_{d}\right)$ belong to $F$ will incur a cost of $\Delta_{t_{j}}=2\left(a_{\imath_{j}}-a_{j t}\right)$, where $\Delta_{\imath}$ means that the adjacent vertex only differs in that component $(l, l)$ is now -1 instead of +1 .

\section{SET COVERING CRITERION}

The optimization problem formulated by Merchant and Rao is as follows $[6]$ :

$$
\begin{gathered}
J(\hat{y})=\operatorname{Min} \sum_{(\imath) \in C}\left(\beta_{\imath \jmath}+\beta_{\jmath \imath}\right)\left(a_{\imath \jmath}-a_{\jmath \imath}\right) y_{\imath \jmath} \\
\sum_{(\imath, \jmath) \in C_{k}} y_{\imath \jmath} \geq 1 \quad k=1,2, \ldots, r \\
y_{\imath \jmath}=0 \text { or } 1 \forall(l, \jmath) \varepsilon C \\
C=\bigcup_{k=1}^{r} C_{k} \text { and } C_{k} \text { is a directed cycle }
\end{gathered}
$$

The $\beta^{\prime}$ s are just weighting coefficients and as long as they are constant (say 1 for simplicity), the following theorem holds. Call (15) with constant $\beta^{\prime}$ s $(15)^{\prime}$.

THEOREM 2. (15) is an equivalent representation of (4) for $\beta_{\imath \jmath}$ constant $\forall(l, \jmath) \in C$.

Proof. (4) is equivalent to (14); hence, it is sufficient to argue that (14) and $(15)^{\prime}$ are equivalent $A s$ Merchant and Rao demonstrated (') the constraints of (15) ensure that all old cycles will be eliminated and no new ones created. From the lemma of the previous section, this is equivalent to moving from $\operatorname{sgn}\left(E_{d}\right)$ to $P^{p}$. In component form, we have $\left[\operatorname{sgn}\left(E_{d}\right)-P^{p}\right]_{\imath_{j}} \neq 0$ if and only if $y_{\imath_{j}}=1$ (or $y_{J^{2}}=1$ ) If $y_{\imath_{\jmath}}=1$ then the contribution towards the above objective function becomes $\Delta_{\imath \jmath}$ (analogously for $y_{\jmath l}$ ) Thus, (14) has become

or

$$
H\left(p^{*}\right)=-2 \operatorname{Mn} \sum_{(i, j) \exists Y_{1 \jmath}=1} \Delta_{\imath \jmath}+E_{d} \operatorname{sgn}\left(E_{d}\right)
$$

$$
J(\hat{y})=\frac{E_{d} \cdot \operatorname{sgn}\left(E_{d}\right)}{2}-\frac{H\left(p^{*}\right)}{2},
$$

(5) [6], Theorem $1, \mathrm{p} 8$ 
which establishes the equivalence since the sense of optimization is opposite for $H$ and $J$.

\section{DISCUSSION}

Although the three criteria are all equivalent, each formulation of the social choice problem has unique characteristics. (2) and (4) are similar in that they are both primal approaches to the problem; both search over the feasible set of $m$ ! permutation matrices, and at each stage of the problem, a feasible solution is known. In contrast, (15) is a dual approach; until the $y_{i j}$ are discovered, no feasible solution to the problem is known.

In (2) an optimal assignment of actions to ranks is determined, while in (4) a search for the optimal matching of a permutation point to a translated election matrix is undertaken. Thus, while both procedures are quadratic assignment problems of a set of "objects" to "positions", the procedures differ by reversing the meanings of objects and positions.

\section{EXAMPLE}

In order to illustrate the three approaches we consider the following example, originally proposed by Condorçet [3]:

\begin{tabular}{|c|c|}
\hline ndividual Ranking & Number with \\
\hline$\left(\begin{array}{lll}a_{1} & a_{2} & a_{3}\end{array}\right)$ & 23 \\
\hline$\left(\begin{array}{lll}a_{2} & a_{3} & a_{1}\end{array}\right)$ & 1 \\
\hline$\left(\begin{array}{lll}a_{2} & a_{1} & a_{3}\end{array}\right)$ & \\
\hline$\left(\begin{array}{lll}a_{3} & a_{2} & a_{1}\end{array}\right)$ & \\
\hline$\left(\begin{array}{lll}a_{3} & a_{1} & a_{2}\end{array}\right)$ & 1 \\
\hline
\end{tabular}

where $\left(\begin{array}{lll}a_{1} & a_{2} & a_{3}\end{array}\right)$ means that alternative $a_{1}$ is preferred to $a_{2}$ and $a_{2}$ is preferred to $a_{3}$. Majority voting leads to an intransitive solution. In order to analyze the problem we construct the following:

$$
\begin{gathered}
A=\left[\begin{array}{ccc}
0 & \frac{33}{60} & \frac{25}{60} \\
\frac{27}{60} & 0 & \frac{42}{60} \\
\frac{35}{60} & \frac{18}{60} & 0
\end{array}\right] \quad E_{d}=\left[\begin{array}{rrr}
0 & \frac{6}{60} & -\frac{10}{60} \\
-\frac{6}{60} & 0 & \frac{24}{60} \\
\frac{10}{60} & -\frac{24}{60} & 0
\end{array}\right] \\
\operatorname{sgn}\left(E_{d}\right)=\left[\begin{array}{rrr}
0 & 1 & -1 \\
-1 & 0 & 1 \\
1 & -1 & 0
\end{array}\right] \frac{E_{d} \cdot \operatorname{sgn}\left(E_{d}\right)}{2}=\frac{2}{3}
\end{gathered}
$$


Since $\sigma_{12}=\sigma_{23} \neq \sigma_{13}$, sgn $\left(E_{d}\right) \notin F$.

Noting that

$$
X=\left[\begin{array}{rrr}
0 & 1 & 1 \\
-1 & 0 & 1 \\
-1 & -1 & 0
\end{array}\right]
$$

we obtain the permutation point :

$$
P^{p^{*}}=\left[\begin{array}{rrr}
0 & -1 & -1 \\
1 & 0 & 1 \\
1 & -1 & 0
\end{array}\right]
$$

which is optimal, implying the social order $\left(\begin{array}{lll}a_{2} & a_{3} & a_{1}\end{array}\right)$.

The optimal permutation matrix is :

$$
\begin{aligned}
P_{p^{*}} & =P_{\bar{p}}=\left[\begin{array}{lll}
0 & 1 & 0 \\
0 & 0 & 1 \\
1 & 0 & 0
\end{array}\right] \\
A_{\bar{p}} & =\left[\begin{array}{ccc}
0 & \frac{42}{60} & \frac{27}{60} \\
\frac{18}{60} & 0 & \frac{35}{60} \\
\frac{33}{60} & \frac{25}{60} & 0
\end{array}\right] .
\end{aligned}
$$

Computing the solutions we have:

$$
\begin{gathered}
\phi(\bar{p})=\frac{42+27+35}{60}=\frac{104}{60} \\
H\left(p^{*}\right)=2 \frac{(-6+10+24)}{60}=\frac{56}{60}=4 \phi(\bar{p})-m(m-1) .
\end{gathered}
$$

From the majority voting solution we obtain the following graph with a cycle :

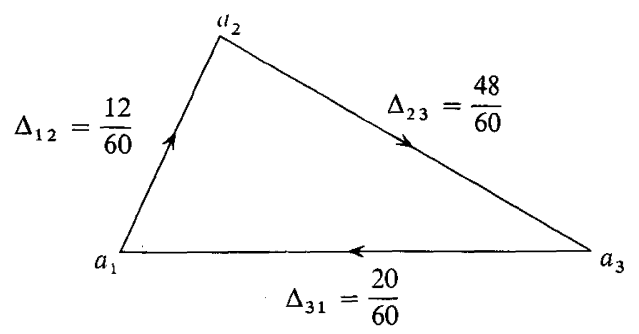

R.A.I.R.O. Reçherche Opérationnelle/Operations Research 
We obtain the set covering problem :

$$
J(\hat{y})=\operatorname{Min} \sum_{(i, j) \in C} 2\left(a_{i j}-a_{j i}\right) y_{i j} \quad C=\{(23),(31),(12)\}
$$

s.t.

$$
\begin{gathered}
y_{23}+y_{31}+y_{12} \geqslant 1 \\
y_{i j}=0 \text { or } 1 \quad \forall(i, \mathrm{j}) \in C
\end{gathered}
$$

The optimal solution is

$$
\begin{aligned}
& \hat{y}_{12}=1 \\
& \hat{y}_{23}=\hat{y}_{31}=0
\end{aligned} \quad \text { and } \quad J(\hat{y})=\frac{1}{5}=\frac{E_{d} \cdot \operatorname{sgn}\left(E_{d}\right)}{2}-\frac{H\left(p^{*}\right)}{2}
$$

which is again equivalent.

\section{REFERENCES}

1. J. M. Blin and A. B. Whinston, Discriminant Functions and Majority Voting, Management Science, Vol. 21, $\mathrm{n}^{\circ}$ 5, 1975, pp. 557-566.

2. V. J. Bowman and C. S. Colantoni, Majority Rule Under Transitivity Constraints, Management Science, Vol. 1, n 9, 1973, pp. 1029-1041.

3. M. CONDORCET, Essai sur l'application de l'analyse à la probabilité de décisions rendues à la pluralité des voix, Paris, 1785.

4. J. Kemeny, Mathematics without Numbers, Daedalus, 1959, 88, pp. 577-591.

5. A. Levenglick, Fair and Reasonable Election Systems, Behavioral Science, Vol. 20, $\mathrm{n}^{\circ} 1,1975$, pp. 34-46.

6. D. K. Merchant and M. R. RaO, Majority Decisions and Transitivity: Some Special Cases, Management Science, Vol. 23, n² 2, 1976, pp. 125-130. 